NOTA CIENTÍFICA

\title{
Índice basado en presiones atmosféricas para la detección de efectos de EI Niño y la Oscilación del Sur frente a la costa peruana
}

\author{
Index based on atmospheric pressures for the detection of EI Niño \\ Southern Oscillation effects off the Peruvian coast
}

\section{Carlos Quispe ${ }^{1}$, Jorge Tam¹, Miguel Saavedra1 e Isaías Gonzáles²}

1 Centro de Investigaciones en Modelado Oceanográfico y Biológico Pesquero (CIMOBP), Instituto del Mar del Perú (IMARPE), Apdo. 22, Lima, Perú. Email Carlos Quispe: cquispe@imarpe.gob.pe

2 Laboratorio Costero de Paita, Instituto del Mar del Perú (IMARPE), Paita, Perú.

Presentado: 25/09/2008 Aceptado: 25/10/2008 Publicado online: 26/02/2009

\section{Resumen}

Se analizó el comportamiento de un índice basado en la diferencia de anomalías de presiones atmosféricas entre Darwin y Paita (IDP), como un indicador de los efectos de El Niño y La Niña frente a la costa peruana, en el Norte del Ecosistema de la Corriente de Humboldt (NECH). La variación temporal a escala mensual del IDP tuvo buena correlación $(r=0,68 ; p<0,01)$ con el Índice de Oscilación Sur (IOS). Se encontró la máxima correlación cruzada $(r=0,59 ; p<0,01)$ entre el IDP y la AN3.4 con un retraso de -3 , lo que permitiría usar el IDP para simular la AN3.4. El comportamiento del IDP obedece a una compleja interacción entre el sistema atmosférico de la región y del Pacifico ecuatorial central.

Palabras clave: Índice, presión atmosférica, El Niño, Oscilación del Sur, Peru.

\section{Abstract}

The behavior of an index based on the difference of atmospheric pressure between Darwin and Paita (IDP) was analyzed, as an indicator of the effects of EI Niño and La Niña in the Northern Humboldt Current Ecosystem (NHCE). The temporal variation of IDP at monthly scale had a good correlation with the Southern Oscillation Index (SOI). Additionally, the maximum cross correlation between IDP and AN3.4 was found at a lag of -3, which could allow to use the IDP to simulate the AN3.4. The behavior of IDP obeys to the complex interaction between the atmospheric system of the region and the Equatorial Pacific.

Keywords: Index, atmospheric pressure, El Niño, Southern Oscillation, Peru.

\section{Introducción}

Los índices oceánicos y atmosféricos son usados para detectar y prever el ciclo El Niño y la Oscilación del Sur (ENOS). Por ejemplo, la NOAA (2003) definió la condición El Niño cuando el Índice Oceánico de El Niño (ONI) sobrepasa el valor $+0,5$ por 3 meses consecutivos, y definió un episodio El Niño cuando el ONI sobrepasa $+0,5$ por 5 meses consecutivos. Por otro lado, el Índice de Oscilación del Sur (IOS) ha sido usado para detectar El Nińo cuando tiene valores negativos, y La Nińa cuando tiene valores positivos (Trenberth 1983).

Sin embargo, también es necesario diseñar índices para detectar y prever el efecto del ciclo ENOS en el Norte del Ecosistema de la Corriente de Humboldt (NECH). Por ejemplo, Purca et al. (2005) han propuesto usar un índice basado en temperaturas superficiales del mar, llamado Índice de Oscilación Peruano (IOP), para detectar los efectos de El Niño en el NECH cuando tiene valores positivos, y efectos de La Nińa cuando tiene valores negativos. En vista de que el IOP está basado en variables oceánicas, y debido a que ENOS es un sistema acoplado océanoatmósfera, se hace necesario diseñar un índice complementario basado en variables atmosféricas. Se hipotetiza que el índice atmosférico tendrá una asociación significativa con el IOS. Por tales motivos, el objetivo del presente trabajo es proponer un índice basado en presiones atmosféricas para detectar los efectos de El Niño en el NECH.

\section{Materiales y métodos}

Datos. Se usaron las siguientes variables para el periodo 2001-2007:

- Índice de oscilación del sur inverso (-IOS), valores mensuales estandarizados obtenidos multiplicando por -1 el IOS de la NOAA (2007). De este modo, valores positivos indican condiciones de El Niño, y valores negativos condiciones de La Niña.

- Anomalía del Índice de temperatura de la Región Niño 3.4 (AN3.4, C), valores mensuales obtenidos de la NOAA (2007).

- Presión atmosférica de Tahití $\left(17,5^{\circ} \mathrm{S}, 149,6^{\circ} \mathrm{W}\right)$ (pT, mbar), valores mensuales obtenidos de la NOAA (2007). La anomalía (aT, mbar) se calculó restando a la presión atmosférica el valor de la media mensual de largo plazo de un periodo base entre 1951 y 1980.

- Presión atmosférica de Darwin $\left(12,4^{\circ} \mathrm{S}, 130,9^{\circ} \mathrm{E}\right)(\mathrm{pD}$, mbar), valores mensuales obtenidos de la NOAA (2007). La anomalía ( $\mathrm{aD}$, mbar) se calculo restando a la presión atmosférica el valor de la media mensual de largo plazo de un periodo base entre 1951 y 1980.

- Presión atmosférica de Paita (pP, mbar), valores mensuales obtenidos de la Sede Regional de Paita para el periodo 20012007. El valor mensual se obtuvo promediando los valores diarios, los cuales a su vez son un promedio de valores horarios obtenidos con un barómetro. La anomalía (aP, mbar) se calculó restando a la presión atmosférica el valor de la media mensual de largo plazo de un periodo base entre 2002 y 2006.

Análisis de datos. Considerando que el IOS es un índice basado en la diferencia de presiones atmosféricas entre Tahití y Darwin (IOS= pT-pD), se analizó el comportamiento de índices alternativos basados en la diferencia de anomalías de presiones atmosféricas entre Tahití y Paita $(\mathrm{ITP}=\mathrm{aT}-\mathrm{aP})$ y entre Darwin y Paita $(\mathrm{IDP}=\mathrm{aD}-\mathrm{aP})$. Antes de calcular las anomalías se sustrajo 1000 mbar a los datos de presiones atmosféricas. Las anomalías se calcularon eliminando el ciclo anual con la siguiente formula:

$$
\mathrm{aP}=\mathrm{mm}-\mathrm{lm}
$$




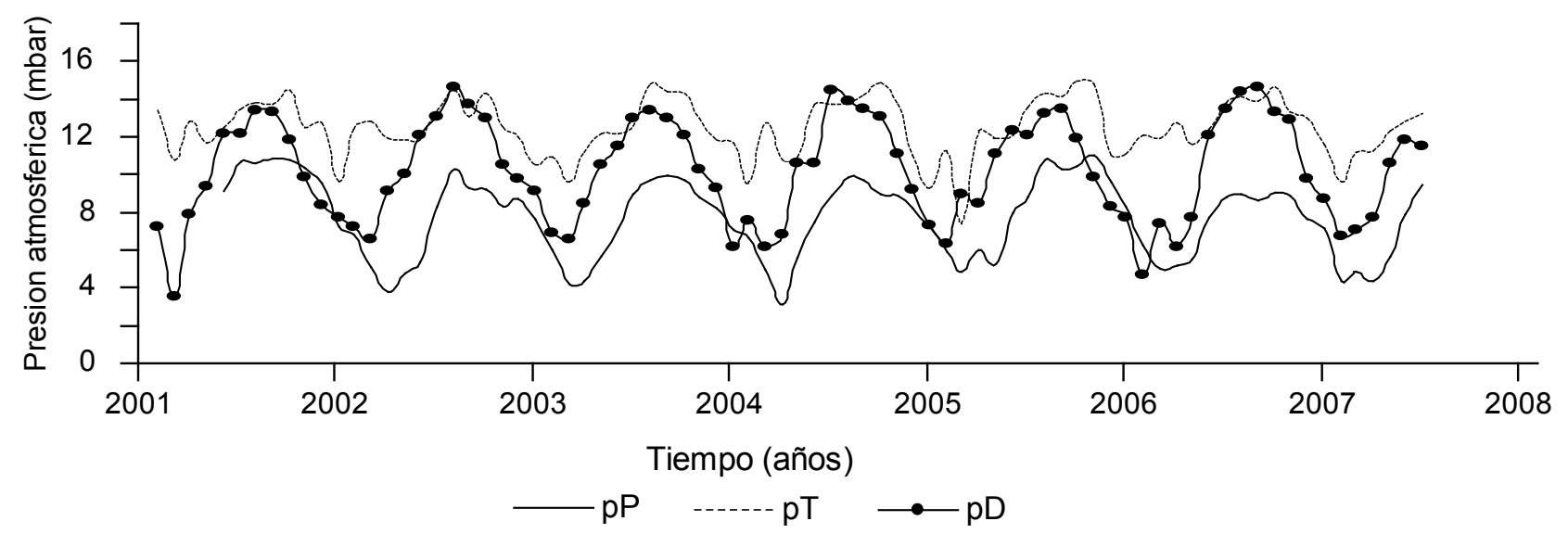

Figura 1 Variación temporal de las presiones atmosféricas pP, pT, pD.

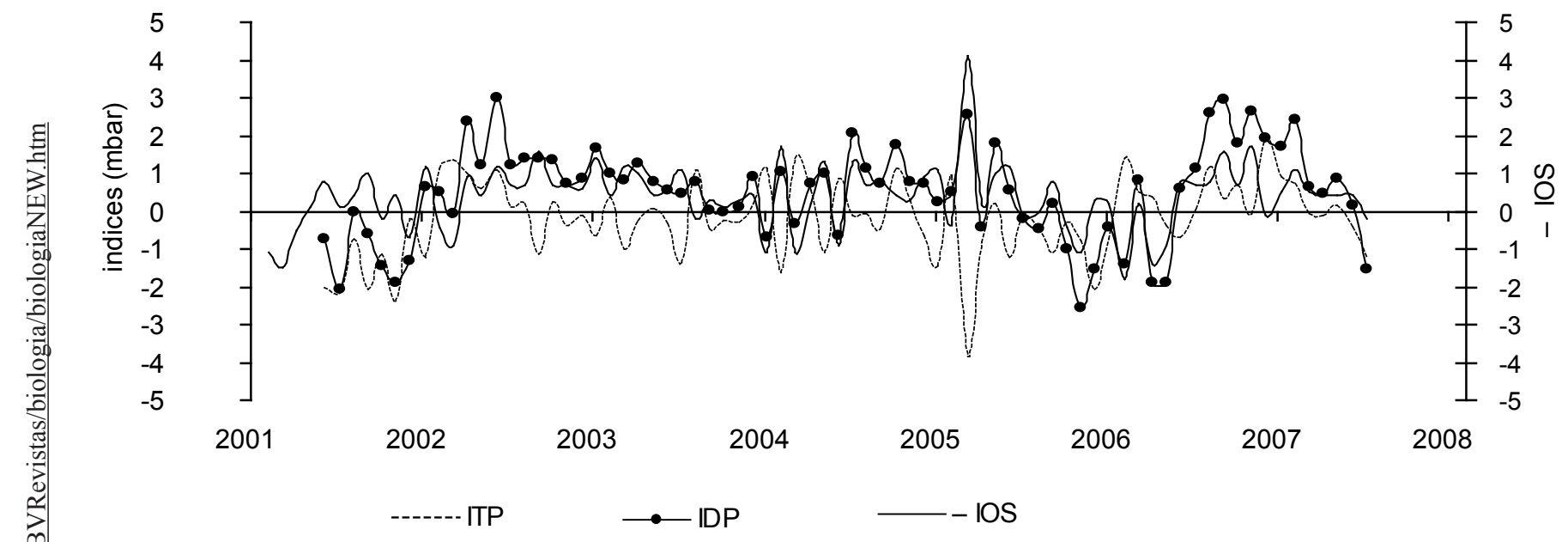

Figura 2. Variación temporal de los indices -IOS, ITP e IDP.

Donde, mm es la media mensual, y $\mathrm{ml}$ es la media mensual de largo plazo (periodo base 2002-2006).

Se calcularon coeficientes de variabilidad modificados $(\mathrm{CVm})$ para comparar series de anomalías con la siguiente fórmula:

$\mathrm{CVm}=100 *$ desviación estándar $/($ media $-\mathrm{min})$

donde media y min son el valor de la media aritmética y el mínimo respectivamente de las series de anomalías que se comparan. Se calcularon correlaciones cruzadas, con retrasos de -4 a +4 meses, entre las variables y los índices potenciales.

Finalmente, se estimó un modelo de regresión lineal simple con retraso, usando como variable predictora el índice basado en presiones atmosféricas y como variable predicha la $A N 3.4$.

\section{Resultados y discusión}

La variación temporal de las presiones atmosféricas $\mathrm{pD}, \mathrm{pT}$ y $\mathrm{pP}$ se muestran en la figura 1. La variación temporal del -IOS, ITP e IDP se muestran en la figura 2. El IDP tuvo un mayor coeficiente de variabilidad $(\mathrm{CV}=27,71 \%)$ que el $-\mathrm{IOS}(\mathrm{CV}=$ $24,87 \%)$ para el periodo $2001-2007$.

Las correlaciones cruzadas entre el ITP y el IDP con el -IOS se muestran en las figuras 3 y 4 . El IDP presentó mayor correlación que el ITP con el -IOS $(\mathrm{r}=0,68 ; \mathrm{p}<0,01)$. Los valores positivos del IDP indican efectos de El Niño (fase cálida del ciclo ENOS), mientras que valores negativos indican efectos de La Niña (fase fría del ciclo ENOS).
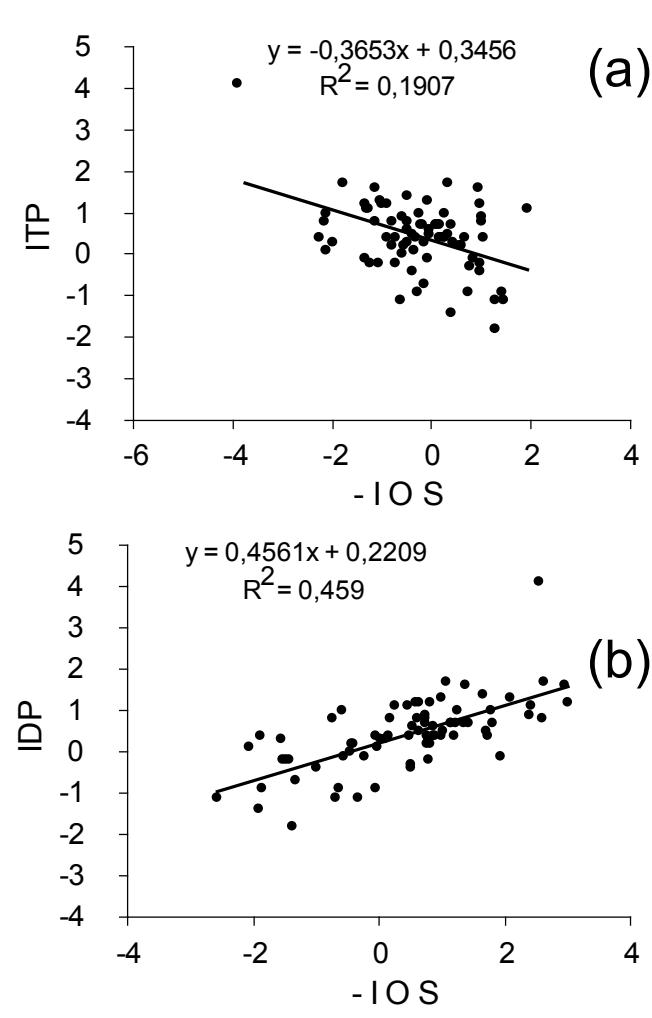

Figura 3. Regresión y coeficiente de determinación entre -IOS vs. (a) ITP y (b) IDP. 


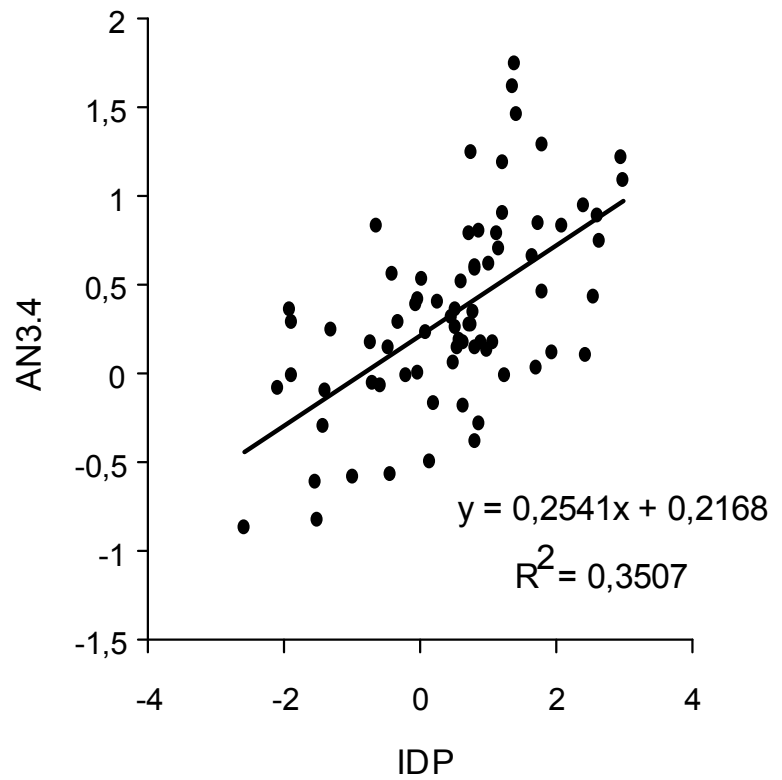

Figura 5. Regresión y coeficiente de determinación de AN3.4 sobre IDP con un retraso de -3 .

La máxima correlación cruzada $(\mathrm{r}=0,59 ; \mathrm{p}<0,01)$ entre el IDP y la $A N 3.4$ se encontró con un retraso de -3, lo que sugirió la factibilidad de estimar por el método de mínimos cuadrados, un modelo de regresión lineal para simular la $A N 3.4$ (Fig. 5):

$$
A N 3.4(\mathrm{t})=0,2541 \operatorname{IDP}(\mathrm{t}-3)+0,2168
$$

Con un $\mathrm{R}^{2}$ de 0,35 ( $\left.\mathrm{p}<0,01\right)$. El modelo presentó un promedio de las desviaciones absolutas (PDA) de $0,31{ }^{\circ} \mathrm{C}$, que representa un $13 \%$ del rango de la $A N 3.4$.

Una aplicación futura de la $A N 3.4$ simulada con el IDP, podría ser su uso como variable independiente en un modelo probabilístico que estime las probabilidades de condiciones trimestrales cálidas, neutras y frías del índice oceánico IOP, por ejemplo el modelo basado en tablas de contingencia (Purca, comunicación personal).

Aunque Paita se encuentra más cerca de Tahití (zona de alta presión) que de Darwin (zona de baja presión) a lo largo de (a)

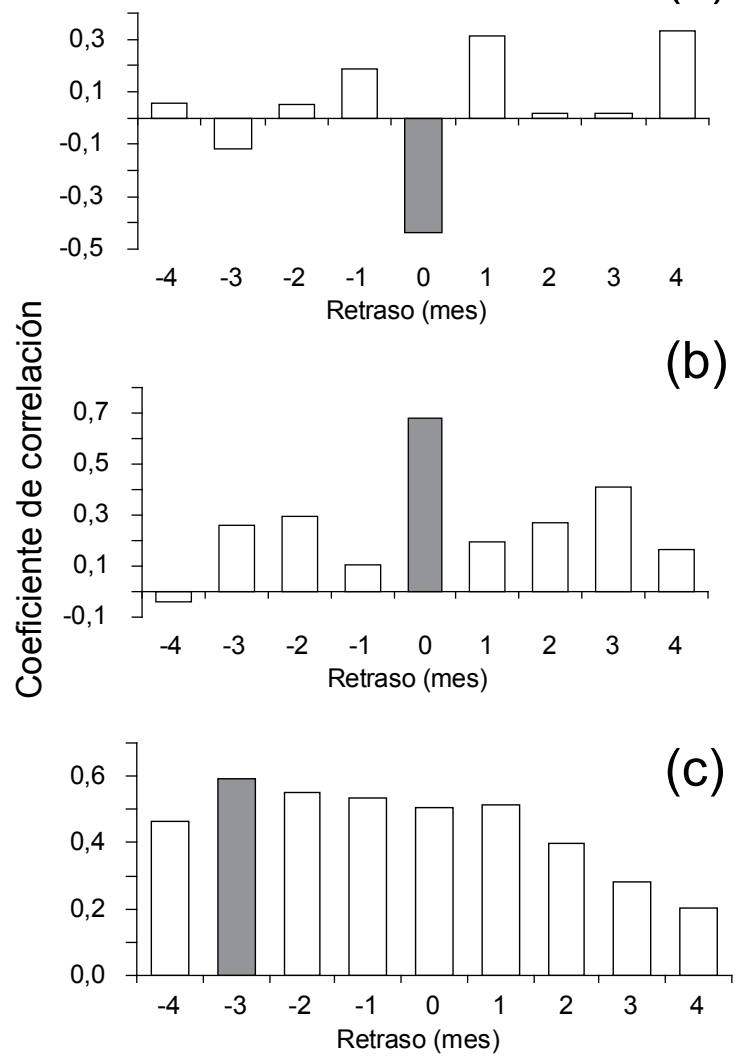

Figura 4. Correlaciones cruzadas entre (a) -IOS vs. ITP, (b) -IOS vs. IDP y (c) IDP vs. AN3.4. La columna sombreada indica la máxima correlación.

la circulación Walker zonal, Paita presenta presiones menores incluso a las de Darwin, lo que sugiere la ubicación de Paita en una celda separada de la celda principal de Walker, como lo sugirió Webster (1983) en Lau y Yang (2003).

\section{Conclusiones}

El IDP mostró una buena correlación con el -IOS ( $\mathrm{r}=0,68$; $\mathrm{p}<$ 0,01 ), con valores positivos indicando efectos de El Niño y valores negativos indicando efectos de La Nińa sobre el NECH.

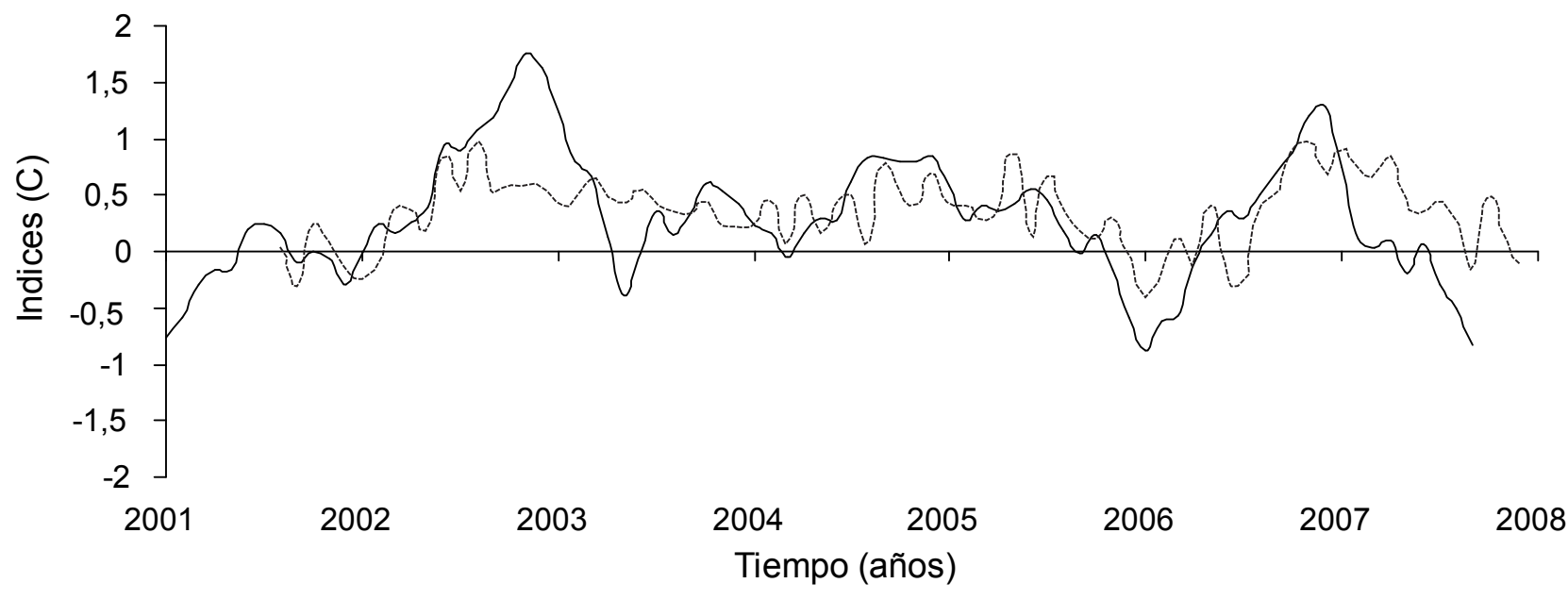

AN3.4 obs

AN3.4 sim

Figura 6. Variación temporal de la AN3.4 observada y la AN3.4 simulada. 
El IDP presentó la máxima correlación cruzada $(\mathrm{r}=0,59 ; \mathrm{p}<$ $0,01)$ con la $A N 3.4$ con un retraso de -3 , permitiendo simular la $A N 3.4$ con 3 meses de anticipación.

En comparación con el IOS, el IDP es un mejor indicador de los efectos de ENOS en el NECH, debido a que está basado en una variable local atmosférica (presión atmosférica de Paita), y presenta un comportamiento que se anticipa a la $A N 3.4$.

\section{Agradecimientos}

Los autores agradecen a Renato Guevara por sus comentarios sobre los índices de ENOS, y a Luis Beltrán del Laboratorio Costero de Paita, por su apoyo en la actualización de los datos de presiones de Paita.

\section{Literatura citada}

Lau, K.-M. and S. Yang. 2003. Walker Circulation. Elsevier Science. pp. 2505-2510.

NOAA. 2007. Monthly atmospheric and SST indices. On-line: http:// www.cpc.ncep.noaa.gov/data/indices.

Purca S., T. Antezana \& R. Riquelme. 2005. The peruvian oscillation index. Capitulo 2. En S. Purca, Variabilidad temporal de baja frecuencia en el Ecosistema de la Corriente Humboldt frente a Perú. Tesis de Doctorado. Universidad de Concepción, Chile.

Trenberth K. E. 1983. Signal versus noise in the Southern Oscillation. Mon. Wea. Rev., 112, 326-332. 